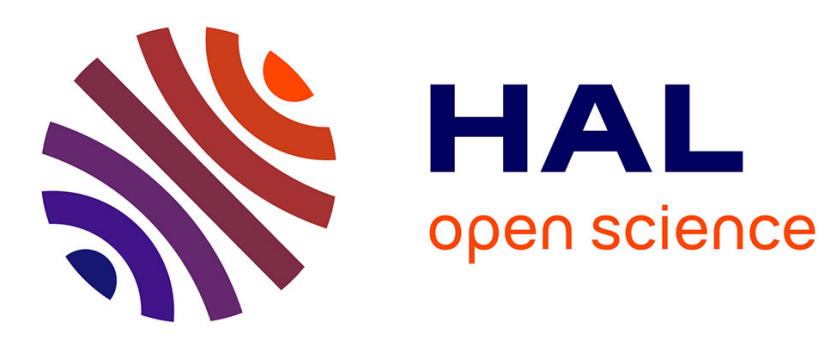

\title{
Zero reflections by a 1D Acoustic Black Hole termination using thermally controlled damping
}

Guillaume Raybaud, Adrien Pelat, Morvan Ouisse, François Gautier

\section{To cite this version:}

Guillaume Raybaud, Adrien Pelat, Morvan Ouisse, François Gautier. Zero reflections by a 1D Acoustic Black Hole termination using thermally controlled damping. Journal of Sound and Vibration, 2021, 510, pp.116282 (9). hal-03359920

\section{HAL Id: hal-03359920 https://hal.science/hal-03359920}

Submitted on 30 Sep 2021

HAL is a multi-disciplinary open access archive for the deposit and dissemination of scientific research documents, whether they are published or not. The documents may come from teaching and research institutions in France or abroad, or from public or private research centers.
L'archive ouverte pluridisciplinaire HAL, est destinée au dépôt et à la diffusion de documents scientifiques de niveau recherche, publiés ou non, émanant des établissements d'enseignement et de recherche français ou étrangers, des laboratoires publics ou privés. 


\title{
Zero reflections by a 1D Acoustic Black Hole termination using thermally controlled damping
}

\author{
Guillaume Raybaud ${ }^{a, 1}$, Adrien Pelat ${ }^{a}$, Morvan Ouisse ${ }^{b}$ and François Gautier ${ }^{a}$ \\ ${ }^{a}$ UMR CNRS 6613 | Laboratoire d'Acoustique de l'Université du Mans I Avenue Olivier Messiaen, 72085 - Le Mans, France \\ ${ }^{b}$ FEMTO-ST institute, Univ. Bourgogne Franche-Comté, CNRS, UMR CNRS 6174 I Department of Applied Mechanics I 24, Chemin de l'épitaphe, \\ 25000 - Besançon, France
}

\section{ARTICLE INFO}

\section{Keywords:}

Enhanced Acoustic Black Holes

Panels vibrations

reflection coefficient

thermal control

\begin{abstract}
A B S T R ACT
The design of lightweight and stiff structures with attractive vibration damping properties is a major issue in mechanical engineering. The insertion of Acoustic Black Holes (ABH) in a beam is a technique that consists in locally reducing its thickness at the end and coating it with a visco-elastic film. The reflection coefficient of the resulting system decreases with frequency and displays typical arches which can exactely reach zero when the amount of losses is well adjusted, reaching the critical coupling condition. However, the precise amount of added damping is very difficult to tune by using classical visco-elastic layers. It is here proposed to control it by using a thermal active system acting in a shape memory polymer (SMP). In this manner, the damping and stiffness profiles become tunable via a given thermal gradient which leads to create an enhanced $\mathrm{ABH}(\mathrm{eABH})$ that can be tuned in real time, so that the critical coupling can be reached. The objective of the paper is to demonstrate numerically and experimentally the capability of an $\mathrm{eABH}$ to achieve exact zero reflections using precise control of temperature gradients.
\end{abstract}

\section{Introduction}

In many applications related to vibro-acoustic engineering, efficient passive control vibration is a central issue for questions of comfort and reliability. It must often be achieved under the constraint of lightening, which means that the proposed vibration mitigation techniques must not increase the mass of the structure. This constraint is generally difficult to take into account because the lighter the structure, the more it is able to vibrate. In this context, new innovative strategies are needed to design light, stiff and damped structures. To satisfy all these conditions, it is necessary to find materials with singular mechanical properties.

The Acoustic Black Hole (ABH), which is currently the subject of extensive research [1, 2, 3, 4], is a response to this challenge. Its usual implementation in a one dimensional (1D) system consists in locally thinning the thickness of a beam according to a power-law profile and coating it with a visco-elastic layer $[5,6]$. Such a termination leads to a trapping effect of the bending waves, which induces an efficient local damping effect. An ABH plays thus the role of an anechoic termination for bending waves. As a consequence, such a $1 \mathrm{D} \mathrm{ABH}$ has a particularly low reflection coefficient $\mathrm{R}$ for propagative flexural waves. It can be shown that the variations of $\mathrm{R}$ with the frequency is a globally decreasing function which exhibits a typical shape in arches. All local drops of the modulus of $\mathrm{R}$ are the consequence of resonances of trapped modes of the $\mathrm{ABH}$, corresponding to modes internally localized [7, 8]. The frequencies and the eigen-shapes of these modes are complex and such characteristics depend both on the coupling between the ABH and the host medium and on the damping mechanisms involved inside the $\mathrm{ABH}$ itself. If particular tuning conditions called critical coupling conditions are achieved, the trapped modes are such that the modulus of $\mathrm{R}$ strictly reaches zero [9]. These critical coupling conditions correspond to the case where the internal losses of the termination (i.e. the active damping mechanisms inside the $\mathrm{ABH}$ ) exactly compensate the energy leakage from the $\mathrm{ABH}$ to the host beam. When these conditions are met, it is said that the ABH is perfectly tuned [10]. In principal, the critical coupling conditions are mode-dependent. Nevertheless, the numerical analysis provided in [7] shows that if one of the first ABH modes is critically coupled then all the other modes are quasi critically coupled. This property explains the efficiency of the $\mathrm{ABH}$, which is seen as a termination having a collection of local resonances conducive to be coupled in a quasi critical way at the same time like the acoustic problem reported in [11] thanks to the intrinsic property gradient due to the thickness profile. The result is a very good broadband performance, which has been observed experimentally

$\operatorname{ORCID}(\mathrm{s})$ :

${ }^{1}$ guillaume.raybaud@univ-lemans.fr 
$[12,13]$. However, precise control of the damping added by a visco-elastic film remains difficult. In practice, the exact properties of the coating material, the gluing conditions, the applied prestresses, and the room temperature are generally poorly known environment settings that strongly affect the resulting damping. As a consequence, it induces a dispersion of the characteristics and precisely tuning the losses leading to critical coupling conditions is therefore tricky but nevertheless necessary to control the quality of the ABH tuning.

The objective of the work reported in this paper is to finely tune the loss profile by means of a temperature gradient along the ABH to fulfill (or not) the critical coupling conditions. This research was inspired, on the one hand, by previous work on smart materials acting on damping $[14,15]$ and on the control of properties by thermal tuning, in particular on shape memory polymers or alloys $[16,17]$. For this purpose, the visco-elastic layer is chosen in order to have high temperature-dependent mechanical properties. The selected material is a tBA/PEGDMA Shape Memory Polymer (SMP). Its use as tunable core in a composite structure and in a metamaterial has been presented respectively in [18] and [19]. Recently, the tBA/PEGDMA material was used as a coating placed on a ABH beam, with a uniform heating of the material resulting in strong variations of its loss factor. Therefore, uniform and adjusted heating allows to control the dissipation mechanisms. Experimental tests associated with numerical simulations show a very clear effect of this thermal control on the amplitude of the reflection coefficient of the ABH [20]. However, the role of the damping on the shape of the arches observed on the reflection properties of the termination, in particular their abilities to reach exactly zero for some particular frequencies, was not described in this work. On the other hand, studies show the interest of controlling the gradient of the intrinsic properties of the $\mathrm{ABH}$. In particular, by questioning the thickness profile $[8,21]$ or by seeking to optimize the damping [22]. In the same direction, the ABH effect has been mimicked [23] or enhanced [24] by means of functionally graded materials. The work presented in this article takes advantage of distributed heaters able to locally control the damping [25], so that loss factors gradients can be obtained. These gradients, which can be tuned in real time, are powerful tools that can be used to follow the poles and zeros of the reflection coefficient in the complex frequency plane. This renders possible the very fine control of the frequency and levels of the arches of the reflection coefficient, allowing in particular the reaching of strictly zero reflection at particular frequencies. The resulting system presented in this paper is called enhanced $\mathrm{ABH}(\mathrm{eABH})$.

The article is organized in three parts. The system is described in the first section. The second section introduces the model of the ABH beam coated with a SMP material. The SMP is exposed to temperature profiles which leads to a parametric analysis and an optimization. Then, the third section presents an experimental validation. Finally, the elements showing the possibility of controlling the reflection coefficient using thermal control are gathered.

\section{Enhanced Acoustic Black Hole using thermal control}

The ABH considered in this paper consists in a tapered aluminium beam following a quadratic profile (see Fig.1.(a)). To avoid a truncation, the tip is extended by a uniform plateau of thickness $h_{t}$ from $x_{t}$ to the end of the beam. This $\mathrm{ABH}$ is enhanced (eABH) by partially covering it with a layer of SMP and an integrated heating device [25, 26].

This heating device is a $150 \mu m$ thick flexible PCB, which enables 5 heating zones $n(n=1,5)$. Each zone contains a temperature sensor and a switch that turns on and off the power in the printed tracks which deliver heat by Joule effect. A micro-controller collects the temperature specified by the user and, according to the temperature measured by the sensors $\left(T_{n}^{\text {Measured }}\right)$, switches on and off the power in the zone. This configuration is convenient to quickly reach the targeted temperature profile $\left(T_{n}^{\text {Targeted }}\right)$ using a PID regulator (see Fig.1.(b) and (c)). However, since the ABH effect occurs in the thinnest part of the beam (where the energy is localized), the effects of the changes in stiffness and damping of the SMP are higher for zones 1 to 3 than for zones 4 and 5. In the following, the discussion is limited to temperature control of zones 1 to 3. Note that a curved shape appears during the ABH manufacturing. The cutting process induces releases of static stresses inside the material, which lead to the slightly bent shape of the ABH, visible in Fig.1.(c). When the ABH is heated, the local temperature variation does not affect significantly the geometry of the tip. It mainly induces a change of the mechanical properties of the polymer (in particular its loss factor), which result in the change in the reflection coefficient.

Two practical limitations restrict the thermal control. First, conversely to SMP, aluminium is a good thermal conductor. The resulting thermal conduction through the beam affects the neighboring zones of the heated one. Practically speaking, if zone $n$ is heated, zones $n-1$ and $n+1$ cannot be more than $20^{\circ} \mathrm{C}$ cooler. Second, the system is vertically clamped. The consequence is that the heat goes up like a flame by thermal convection. This phenomenon affects therefore the upper zones. In addition to the thermal conduction, if zone $n$ is heated, the zone $n-1$ cannot be more than $10^{\circ} \mathrm{C}$ cooler. To summarize, the temperature in a given zone depends on the temperature of the others. For example, 
(a)

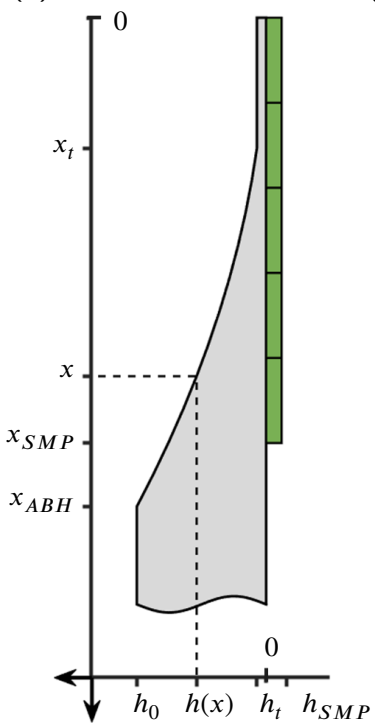

(b)

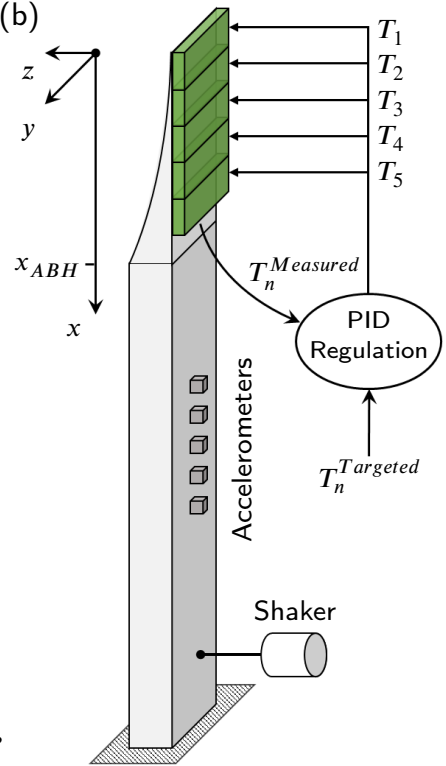

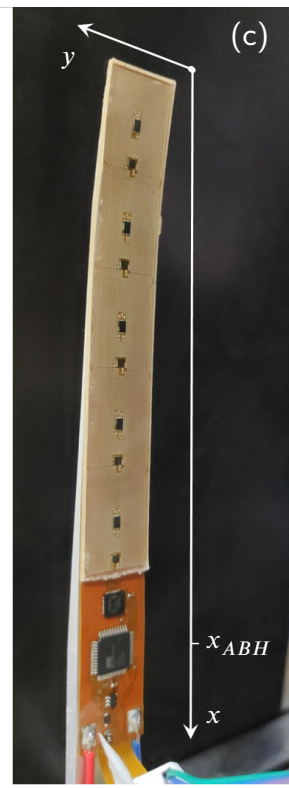

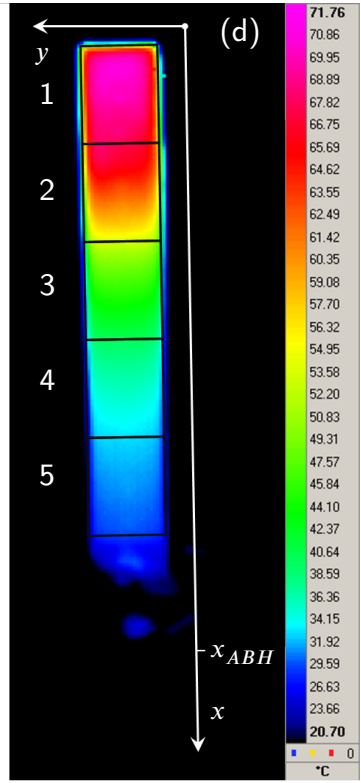

Figure 1: (a) Geometric parameters of the eABH: $h_{0}=5 \mathrm{~mm}, b=20 \mathrm{~mm}$ (width of the beam), $x_{A B H}=150 \mathrm{~mm}, h_{t}=0.089 \mathrm{~mm}$, $x_{t}=20 \mathrm{~mm}, h_{S M P}=2 \mathrm{~mm}, x_{S M P}=130 \mathrm{~mm}, h(x)=h_{t}, \forall x \in\left[0, x_{t}\right] ; h(x)=h_{0}\left(x / x_{A B H}\right)^{2}, \forall x \in\left[x_{t}, x_{A B H}\right] ; h(x)=h_{0}, \forall x>$ $x_{A B H}$. (b) Schematic diagram of the experimental set-up, $T_{n}$ is the notation for the temperature of the zone $n$. (c) Technical realization of the thermal control device of the $\mathrm{ABH}$. (d) Typical temperature field measured on the eABH.

if only zone 3 is heated up to $70^{\circ} \mathrm{C}$, then the temperature of $T_{1}, T_{2}, T_{3}, T_{4}$ and $T_{5}$, will roughly be $(50,60,70,50$, $30)^{\circ} \mathrm{C}$ and not $(20,20,70,20,20)^{\circ} \mathrm{C}$. These practical limits and the reachable configurations can be represented in the parameters space displayed in Fig.2. The minimum temperature is chosen at $30^{\circ} \mathrm{C}$. Below this temperature, the material properties are almost independent of temperature with a low loss factor [18]. The maximum is chosen at $70^{\circ} \mathrm{C}$ because it approaches the glass transition temperature of the SMP (at $100 \mathrm{~Hz}$ ), where the loss factor reaches its maximum value. The green volume shown in Fig. 2 represents all the configurations which can be reachable. Points 1 to 6 will be used later as specific measurements configurations. The plane represented in brown corresponds to the simulation plane used in section 3.3.

\section{Numerical analysis}

\subsection{Reflection matrix of an eABH}

The reflection coefficient $\mathrm{R}$ of the termination is an important feature for the $\mathrm{eABH}$, which can be computed using the Impedance Matrix Method [27]. In harmonic regime (the time factor $e^{j \omega t}$ is omitted for brevity, $\omega$ being the circular frequency), and in the framework of the Euler Bernoulli model, the bending vibrations of a non-uniform beam can be modeled by a state equation:

$$
\frac{d \mathbf{X}}{d x}=\mathbf{H X}
$$

where $\mathbf{X}$ is the state vector composed of the bending displacement, the slope, the shear force, and the bending moment. The transition matrix $\mathbf{H}$ is given by

$$
\mathbf{H}=\left[\begin{array}{cc|cc}
0 & 1 & 0 & 0 \\
0 & 0 & 0 & 1 / E(T) I \\
\hline-\rho S \omega^{2} & 0 & 0 & 0 \\
0 & 0 & -1 & 0
\end{array}\right]=\left[\begin{array}{ll}
\mathbf{H}_{1} & \mathbf{H}_{2} \\
\mathbf{H}_{3} & \mathbf{H}_{4}
\end{array}\right],
$$

where $\rho$ is the mass density, $S$ the cross-sectional area, $I$ the moment of inertia. The modulus $E(T)$ is the local equivalent Young's modulus of the sandwich beam compound by the aluminium and SMP layers at temperature $T$. 


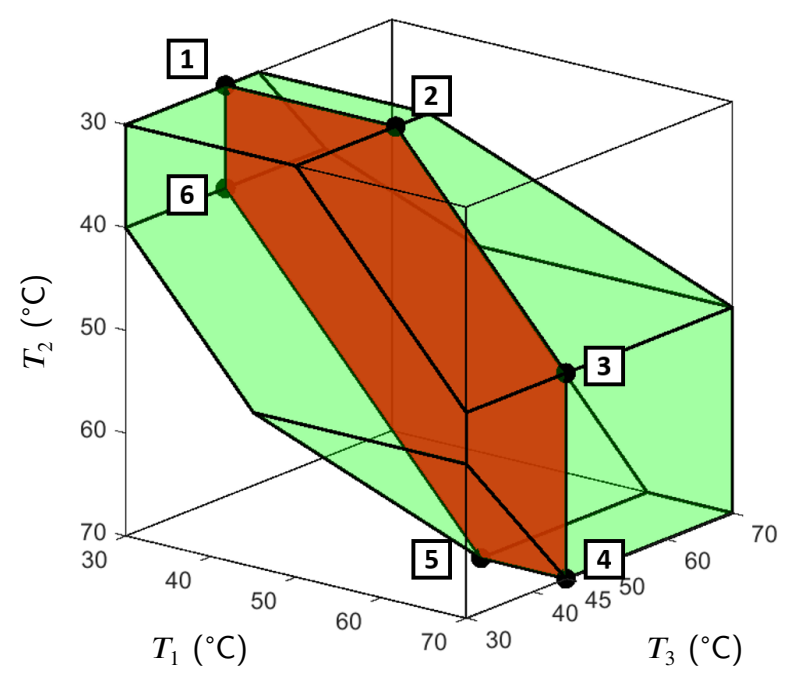

Figure 2: The green volume is the parameter space describing the possible values of the temperatures $T_{1}, T_{2}$ and $T_{3}\left({ }^{\circ} \mathrm{C}\right)$. The brown area is the plane corresponding to $T_{3}=45^{\circ} \mathrm{C}$. This area is delimited by the configurations numbered from 1 to 6 defined by a set of 3 temperatures $\left(T_{1}, T_{2}, T_{3}\right)$ : configuration \#1 $(30,30,45)^{\circ} \mathrm{C}$, configuration \#2 $(50,30,45)^{\circ} \mathrm{C}$, configuration \#3 $(70,50,45)^{\circ} \mathrm{C}$, configuration \#4 $(70,70,45)^{\circ} \mathrm{C}$, configuration \#5 $(60,70,45)^{\circ} \mathrm{C}$, configuration \#6 $(30$, $40,45)^{\circ} \mathrm{C}$.

The equivalent modulus $E(T)$ is computed in Eq. (2) using the Ross Ungar Kerwin mixing law (see [12], Eq. (24), p.76). To simplify the numerical model, the imposed temperature field is supposed to be uniform by zone, which is compatible with the thermal state of the beam described in section 2. The relationship between the complex Young's modulus of the SMP and the temperature is given in details in [18], Eq. (3) p.402 and the mechanical parameters used in our simulations are the ones given this reference.

The influence of the PCB used for the heating system, its electronics elements and the glue are ignored in the modeling. The impedance matrix $\mathbf{Z}$, that relates the kinematic and force components of the state vector $\mathbf{X}$, i.e. $\left[\begin{array}{ll}F & M\end{array}\right]^{\mathrm{T}}=\mathrm{j} \omega \mathbf{Z}[w \theta]^{\mathrm{T}}$, obeys the Riccati equation:

$$
\frac{\partial \mathbf{Z}}{\partial x}=-\mathbf{Z} \mathbf{H}_{1}-j \omega \mathbf{Z} \mathbf{H}_{2} \mathbf{Z}+\frac{\mathbf{H}_{3}}{j \omega}+\mathbf{H}_{4} \mathbf{Z} .
$$

The impedance $\mathbf{Z}$ for $0<x \leq x_{A B H}$ is obtained using a numerical integration from the boundary $x=0$ where its value is imposed by the free end condition $\mathbf{Z}(0)=0$. Boundary conditions at the other end have no influence on $\mathbf{Z}$. The integration is performed using a Magnus scheme. At the end of the $\mathrm{ABH}$, at $x_{A B H}$, the reflection matrix $\mathbf{R}$ is computed from $\mathbf{Z}$ and the matrix $\mathbf{E}=\left[\mathbf{E}_{1} \mathbf{E}_{2} ; \mathbf{E}_{3} \mathbf{E}_{4}\right]$, whose columns are the eigen-vectors of $\mathbf{H}$. The reflection matrix $\mathbf{R}$ is given by (see [27])

$$
\mathbf{R}=\left[j \omega \mathbf{Z} \mathbf{E}_{2}-\mathbf{E}_{4}\right]^{-1}\left[\mathbf{E}_{3}-j \omega \mathbf{Z} \mathbf{E}_{1}\right] .
$$

In this paper, the reflection coefficient named R corresponds to the term associated to propagative waves of $\mathbf{R}\left(\mathbf{R}_{11}\right)$ [12].

\subsection{Zeros of $R$ in the complex frequency plane: case of an $A B H$ with uniform temperature}

For the eABH at room temperature $\left(20^{\circ} \mathrm{C}\right.$, see Fig.3.(a)), the SMP coating is stiff and weakly dissipative. In this case, the $\mathrm{ABH}$ effect is not visible and the reflection coefficient $\mathrm{R}$ is close to 1 (see Fig.3.(b)). The magnitude of $\mathrm{R}$ is mapped in the complex frequency plane using a logarithmic scale $(20 \log |\mathrm{R}|)$ in the background of Fig.3.(c). The blue and yellow spots correspond respectively to the zeros and the poles of $\mathrm{R}$, the latter being associated to the trapped modes localized in the ABH. These particular points are symmetrically located on both sides of the real frequency axis (highlighted in red), when the system is conservative, which is nearly the case at $20^{\circ} \mathrm{C}$. By zooming around the first 

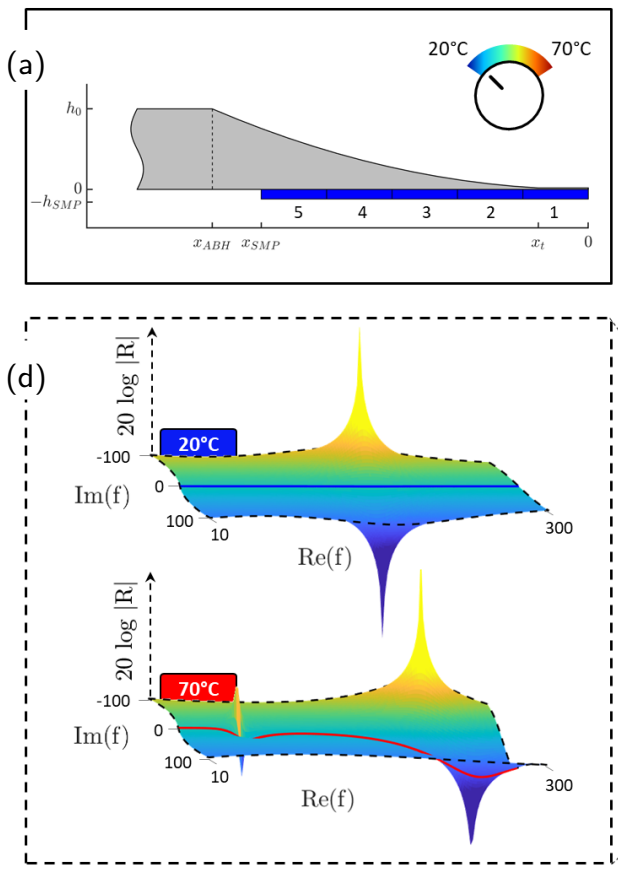

(b)

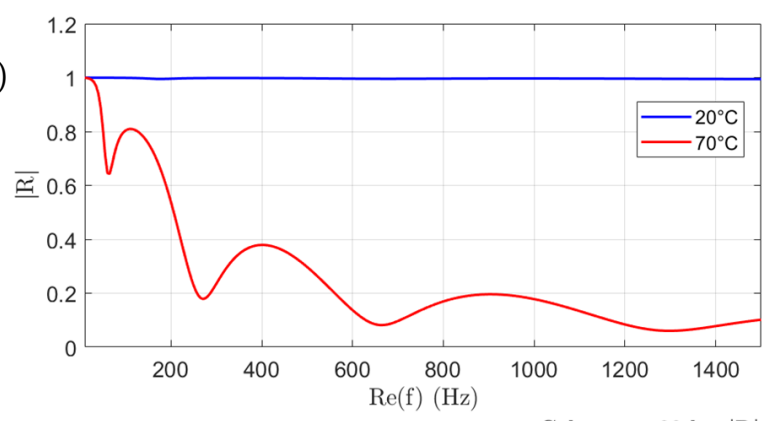

(c)

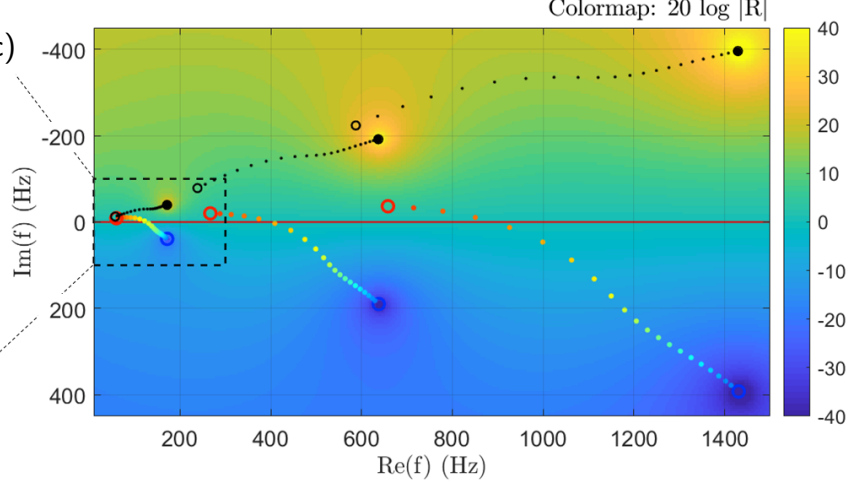

Figure 3: (a) Schematic diagram of the eABH uniformly heated to $20^{\circ} \mathrm{C}$. (b) $|\mathrm{R}|$ as function of the real frequency. (c) The color mapping corresponds to the magnitude of the reflection coefficient plotted in the complex frequency plane at $20^{\circ} \mathrm{C}$ using a logarithmic scale $(20 \log |\mathrm{R}|)$. For a given temperature, the locations of the poles and zeros are indicated by markers. The correspondence between the markers' color and the temperature is defined by the colorbar of Fig.3.(a). (d) Zoom of $|\mathrm{R}|$ in the complex frequency plane, in the vicinity of the first pair of zero and pole. The blue (resp. red) curve denotes the variations of $20 \log |R|$ versus $\operatorname{Re}(f)$ at $20^{\circ} \mathrm{C}$ (resp. $70^{\circ} \mathrm{C}$ ).

pair of zero and pole (see the zoom area in Fig.3.(d)), it is seen that their influences compensate one to each other due to symmetry, and so $|\mathrm{R}|$ remains close to 1 along the real frequency axis.

Another state of the $\mathrm{ABH}$ is considered which consists in a uniformly heated termination $\left(T=70^{\circ} \mathrm{C}\right)$. The system is no longer conservative since the SMP layer becomes a very effective damping layer. In this case, the typical variation in arches of $\mathrm{R}$ is observed (see Fig.3.(b)). By looking at the complex frequency plane, the set of poles and zeros, represented by the black and red circle markers respectively, are now asymmetrically arranged (see Fig.3.(c)). By zooming around the first pairs of zeros and poles (see Fig.3.(d)), it is seen that they do not compensate anymore, which causes $|\mathrm{R}|$ (red curve) to fall into the zeros' pits (see Fig.3.(d)).

Through this analysis, the arches of $|R|$ result from the presence of zeros in the complex frequency plane. By heating the eABH uniformly, the zeros are basically moved towards the real frequency axis, so $|\mathrm{R}|$ is reduced. By following their trajectory during warming up (see Fig.3.(c), gradient color markers), it can be seen that the zeros cross the real frequency axis at a given temperature. This indicates that the critical coupling conditions are fulfilled. The $\mathrm{ABH}$ effect is maximum in this case since strictly no reflection is induced: the $\mathrm{ABH}$ configuration is perfectly tuned at this frequency and the $\mathrm{ABH}$ behaves as a perfect wave trap. This phenomenon is nevertheless highly localized since the critical coupling conditions for a given mode are fulfilled only for one heating temperature and a single frequency.

\subsection{Zeros of $R$ in the complex frequency plane: case of an $A B H$ with a temperature gradient}

The critical coupling conditions for a given mode of the ABH can be obtained by using a uniform temperature field. The frequency for which this phenomenon occurs is fixed by the ABH configuration and cannot be chosen a priori. Adding degrees of freedom to the system is a way to permit this frequency tuning. This is performed by imposing a heating temperature gradient, instead of a uniform heating temperature. The resulting $\mathrm{ABH}$ is an $\mathrm{ABH}$, for which a frequency tuning is possible on a wide range. 


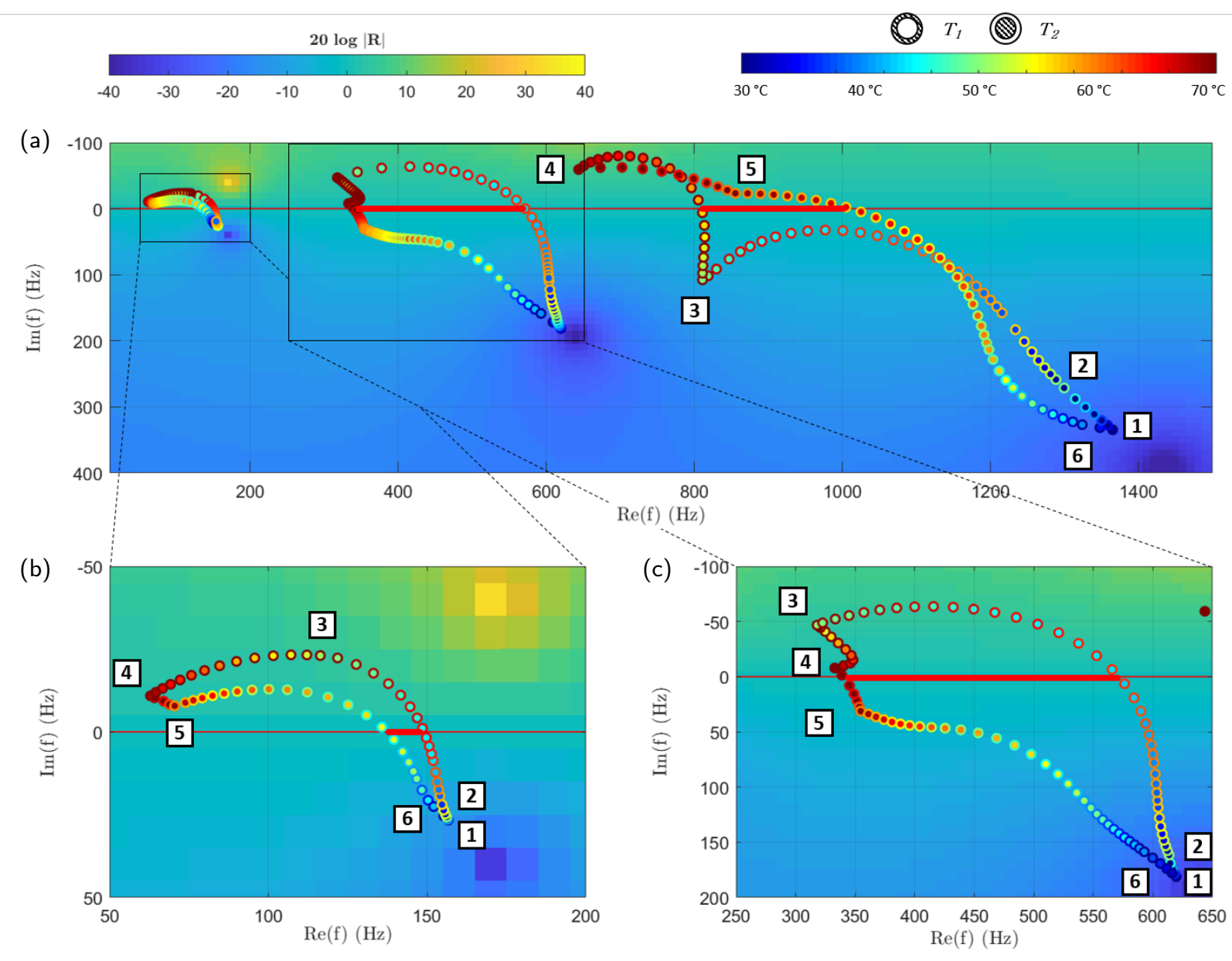

Figure 4: (a) Trajectories of the zeros following the parameter plane (see Fig.2). The bi-color markers indicate the temperature $T_{1}$ and $T_{2}$ of each configuration. The background color corresponds to the $20^{\circ} \mathrm{C}$ configuration. (b) Focusing on the first zero. (c) Focusing on the second zero.

In practice, the temperature gradient is realized by using a multi-zone heating device. Five independent heating sources are implemented in the ABH termination (see Fig.1). The number of zones is chosen in order to create the temperature gradient while keeping a reasonable degree of technological complexity. A numerical investigation of the influence of the multi-zone heating system is provided in Fig.4. The values of the temperature used for the control are those specified by the brown plane of Fig.2 (points 1 to 6).

Zones 1 and 2 are the zones whose temperatures have the most influence on the positions of the poles. To simplify the analysis, the values of $T_{3}, T_{4}$ and $T_{5}$ are fixed at $45^{\circ} \mathrm{C}, 36^{\circ} \mathrm{C}$ and $31^{\circ} \mathrm{C}$ respectively. $T_{1}$ and $T_{2}$ are chosen as the key parameters of the analysis and follow the cycle described by points 1 to 6 in the work space given in Fig.2.

The trajectory of a given zero during this cycle draws the perimeter of a domain whose all internal points are accessible using a pair $\left(T_{1}, T_{2}\right)$ located in the brown zone of the workspace (see Fig.2). The trajectories of the 3 first zeros are depicted in Fig.4. Their shapes depend on 2 combined effects due to the SMP layer: the material becomes 1/ softer and $2 /$ more damped as the temperature increases. A remarkable point is that domains inside the trajectories of the first 3 modes cover a large part of the real frequency axis. This is highlighted by red segments in Fig.4. The critical coupling conditions can be fulfilled for any point on these red segments: for mode 1, the critical coupling conditions can be reached if $f \in[135-150 \mathrm{~Hz}]$; for mode 2 if $f \in[340-570 \mathrm{~Hz}]$; for mode 3 if $f \in[810-1090 \mathrm{~Hz}]$. Other parametric studies, not reported in the paper, show that temperatures $T_{3}$ to $T_{5}$ have minor effects on the positioning of the zeros. This is due to the fact that these heating points are located in areas where the SMP layer has little influence 
due to the large thickness of the beam. Finally, a system with equivalent control could have been an enhanced $\mathrm{ABH}$ with only the first two heating zones at the beam tip, without the other three [20].

This parametric study shows that a temperature gradient obtained with 2 heating points allows the positioning of a zero of the system so that the critical coupling conditions are fulfilled. In other words, independently of the temperature of the last 3 zones, it is possible to find a pair $\left(T_{1}, T_{2}\right)$ allowing to reach these conditions. The frequency for which these critical coupling conditions are obtained is tunable using the $T_{1}$ and $T_{2}$ parameters over a wide frequency range. In order to increase the total trapping effect of the $\mathrm{ABH}$ (i.e. to increase the frequency range over which $|\mathrm{R}|$ is low), the conditions for which several modes can be critically coupled at the same operating temperature are investigated in the following.

\subsection{Achieving single and double critical coupling conditions}

For each pair $\left(T_{1}, T_{2}\right)$ of the control temperatures, the variations versus frequency of $|\mathrm{R}|$ have local minima leading to drops. Fig.5 reports a parametric study describing these variations. First, the conditions for reaching the critical coupling for mode 3 are discussed. The value of the minimum of the 3 rd drop is denoted $V_{3}$ and is equal to zero when the critical coupling conditions are satisfied. $V_{3}$ is plotted in Fig.5.(a) as a function of $\left(T_{1}, T_{2}\right)$. The blue valley, whose bottom is highlighted by a white line, corresponds to the minimum of $V_{3}$. Each point of the line corresponds to a pair $\left(T_{1}, T_{2}\right)$, giving rise to the critical coupling conditions. For each of these points the variations of $|\mathrm{R}|$ are plotted in Fig.5.(b). The result is a curve bundle, showing all configurations for which the critical coupling conditions are satisfied for mode 3. In practice, the red segment in Fig.5.(b) shows that the critical coupling conditions for mode 3 can be obtained for any targeted frequency $f \in[810-1090 \mathrm{~Hz}]$ if the right pair $\left(T_{1}, T_{2}\right)$ is used.

Among all the curves of the bundle, the bold black curve is the one with the lowest averaged value of $\mathrm{R}$. This average is computed over the frequency band $[10-1500 \mathrm{~Hz}]$. Note that the pair $\left(T_{1}, T_{2}\right)$ corresponding to this particular case is indicated by a black dot in Fig.5.(a).

Now the procedure is generalized for the 3 first drops. The objective is to try to obtain the critical coupling conditions for several modes simultaneously. To do so, the absolute minimum of $|\mathrm{R}|$ over the whole frequency range is searched for. For a given pair $\left(T_{1}, T_{2}\right)$, this minimum is denoted $V_{\min }$ and indicates the existence of a critical coupling for at least one of the modes 1,2 or 3 . The location of the $V_{\min }$ points are indicated by white lines 1,2 and 3 in Fig.5.(c). Line 3 is the one already identified in Fig.5.(a). The intersections between these 3 lines, orange and green dots, indicate the temperature conditions leading to multiple critical coupling conditions. The orange configuration leads to a critical coupling of modes 1 and 2, while the green one corresponds to modes 2 and 3 (see Fig.5.(d)). It appears that double critical coupling conditions can be reached. Exact triple critical coupling conditions cannot, with the studied configuration. However, a good compromise is already found for the orange configuration where two drops reach zero and the third one almost reaches zero as well.

The map 5.(c) is a synthetic representation of the temperature pairs that can give rise to simple, double and quasi triple critical coupling conditions. 
(a)
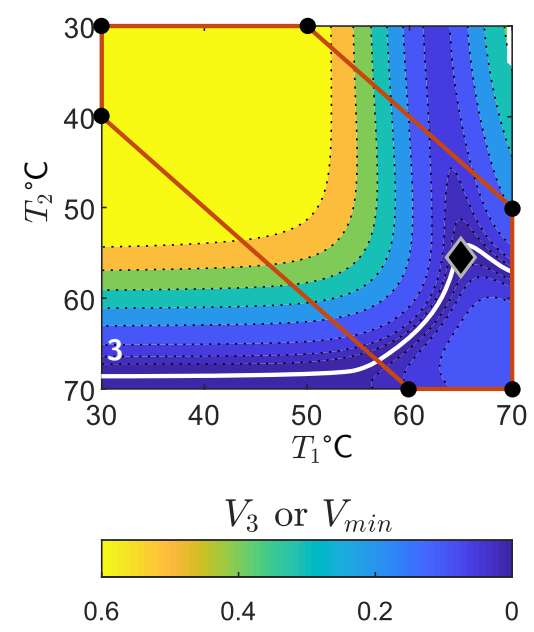

(c)

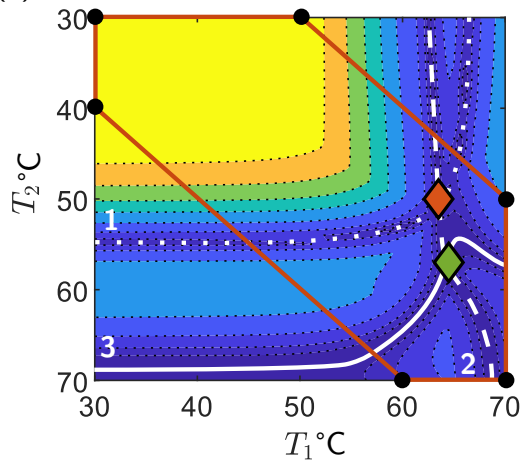

(b)

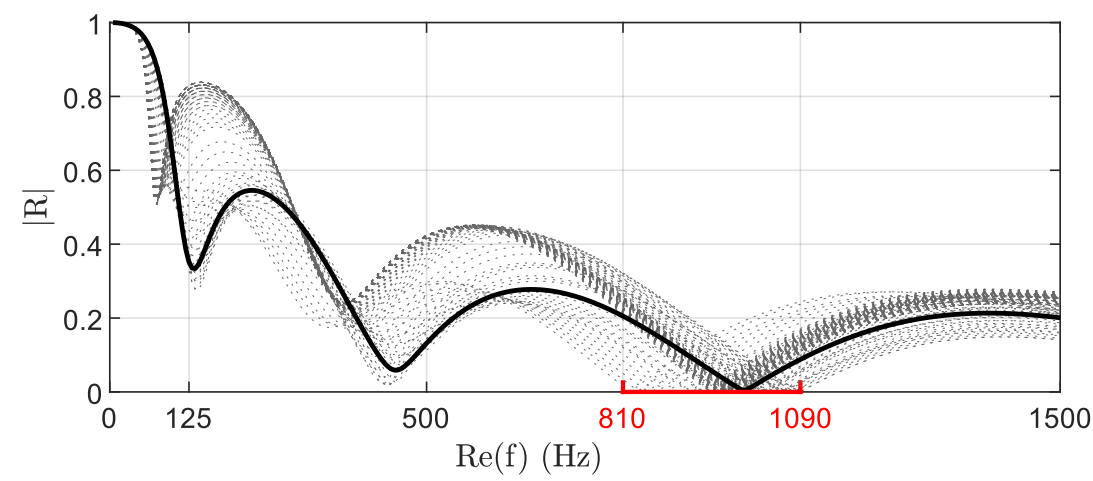

(d)

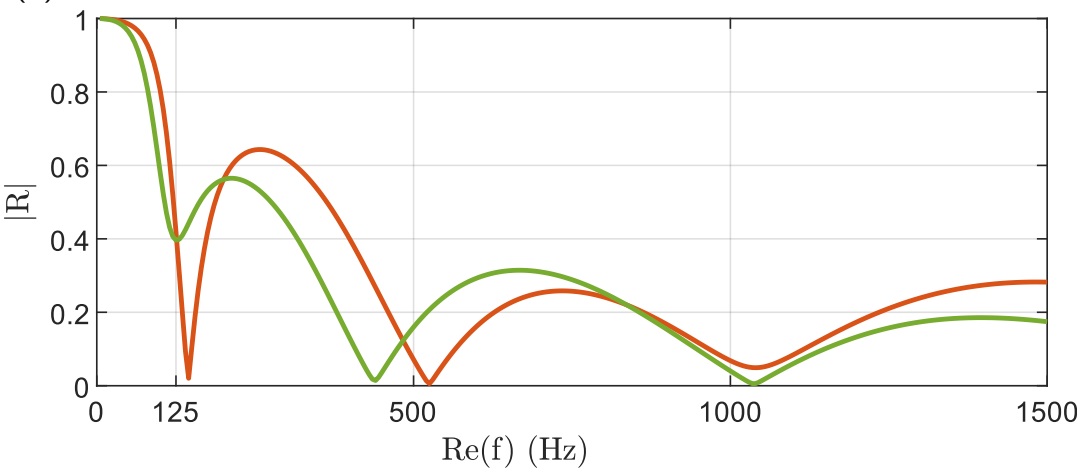

Figure 5: (a) Variations of $V_{3}$ versus $T_{1}$ and $T_{2}, V_{3}$ being the local minimum value of $\mathrm{R}$ in the vicinity of the resonance frequency of mode 3. The brown polygon demarcates the parameter plane $\left(T_{1}, T_{2}\right)$ defined in Fig.2. The white line is the loci of the minimum values of $V_{3}$, which give rise to critical coupling conditions. (b) Each curve of the bundle corresponds to the variations of $|\mathrm{R}|$ versus frequency for the temperatures pairs $\left(T_{1}, T_{2}\right)$ belonging to the white line of (a). The black bold curve is associated to case having the lowest averaged value over the whole frequency range. The corresponding control temperatures are indicated by the black marker in (a). The red segment indicates the reachable critical coupling conditions. (c) Variations of $V_{\min }$ versus $T_{1}$ and $T_{2}, V_{\min }$ being the minimum value of $|R|$ over the whole frequency range. Green and orange diamond markers indicate two specific configurations leading to double critical coupling conditions. (d) Variations of $|\mathrm{R}|$ versus frequency for $T_{1}=63.5^{\circ} \mathrm{C}, T_{2}=50^{\circ} \mathrm{C}$ (orange diamond marker in (c)) and for $T_{1}=64.5^{\circ} \mathrm{C}$, $T_{2}=57^{\circ} \mathrm{C}$ (green diamond marker in (c)) 
(a)

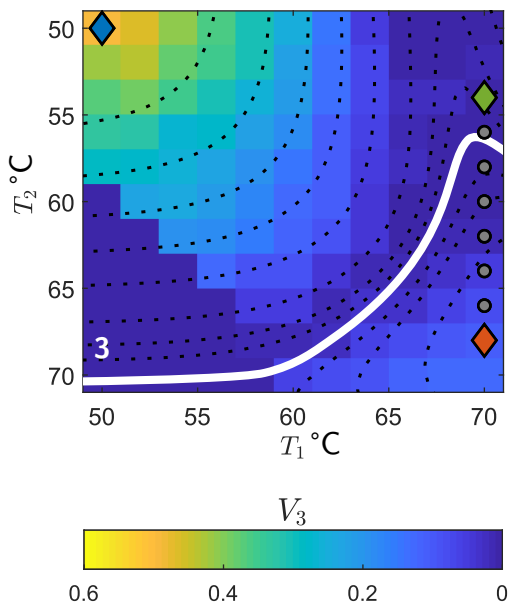

(b)

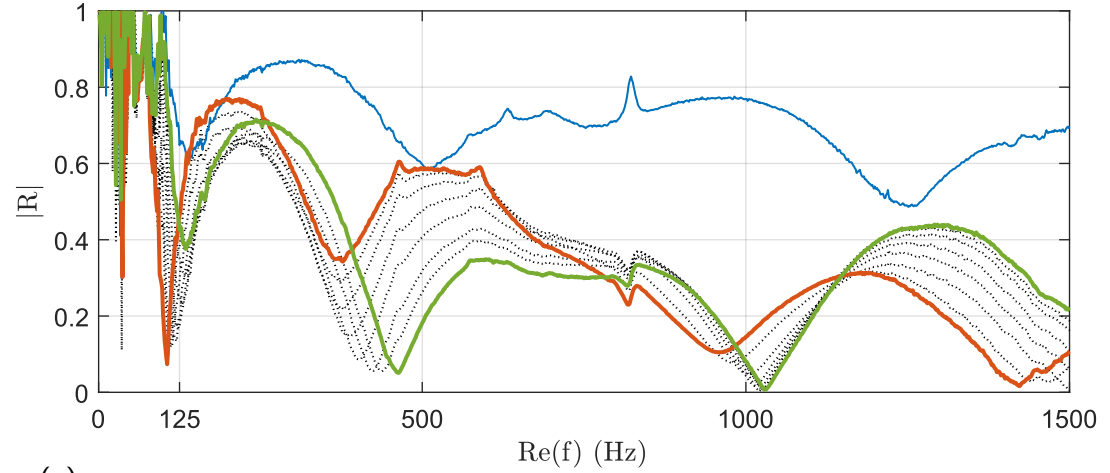

(c)

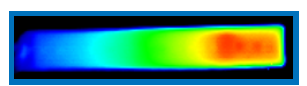

$\left(50^{\circ} \mathrm{C}, 50^{\circ} \mathrm{C}\right)$

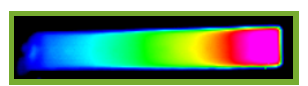

$\left(70^{\circ} \mathrm{C}, 54^{\circ} \mathrm{C}\right)$

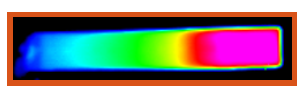

$\left(70^{\circ} \mathrm{C}, 68^{\circ} \mathrm{C}\right)$

Figure 6: (a) Measured variations of $V_{3}$ versus $T_{1}$ and $T_{2}, V_{3}$ being the minimum value of $\mathrm{R}$ in the vicinity of the resonance frequency of mode 3. White line and black dotted lines are the iso-value lines reported from the simulation (see Fig.5.(a)). (b) Measured variations of $\mathrm{R}$ for 3 particular configurations indicated in (a): blue point $\left(T_{1}=50^{\circ} \mathrm{C}, T_{2}=50^{\circ} \mathrm{C}\right)$, green point $\left(T_{1}=70^{\circ} \mathrm{C}, T_{2}=54^{\circ} \mathrm{C}\right)$, orange point $\left(T_{1}=70^{\circ} \mathrm{C}, T_{2}=68^{\circ} \mathrm{C}\right)$. The gray bundle corresponds to the gray markers between the green and orange ones. (c) thermal maps associated to the 3 selected configurations given in (b).

\section{Experimental validation}

The measurement set-up (see Fig.1) consists of a shaker (LDS V201) with an impedance head (PCB 288D01) which excites the base of the clampled $\mathrm{ABH}$ beam with a white noise covering the frequency range of interest. The displacement field is measured using 5 accelerometers (B\&K 4394) $2.5 \mathrm{~cm}$ apart located midway between the shaker and the $\mathrm{ABH}$ termination. Then, the contributions of the forward and backward propagating and evanescent waves amplitudes are identified using an inverse technique [12]. From there, $\mathrm{R}$ is estimated.

A large number of $\left(T_{1}, T_{2}\right)$ configurations have been tested (see Fig.6.(a)) in order to reproduce the map given in Fig.5.(a). Some features of the thermal control of damping are only partially accounted for in the modeling, as well as the influence of the adhesive at the interface. In practice, the temperature field is not uniform (see Fig.1.(d)), so an average temperature per zone captured by the thermal camera is considered. In addition, the material is hotter in contact with the heating device located at the interface with the beam, whereas the camera measures the temperature at the surface. This is why a small correction of the temperatures used in the simulation is taken into account to improve the model fit: the simulated results are given for $T_{1}^{\text {simu }} \simeq T_{1}^{\exp }+4$ and $T_{2}^{\text {simu }} \simeq T_{2}^{\exp }+2$. With these assumptions, the obtained model allows a fine analysis of the thermal control of the ABH. However, a discrepancy with the experimental study can be expected.

Even if they do not coincide, experimental and numerical results show similar trends: in Fig.5.(a), the blue valley corresponds to the minimum of $|\mathrm{R}|$, and has the same shape as the simulations provided in the previous paragraph. The white line from Fig.5.(a) is reported in Fig.6.(a), showing that the model correctly predicts the $\left(T_{1}, T_{2}\right)$ pairs leading to strict or quasi critical coupling conditions.

Three particular pairs $\left(T_{1}, T_{2}\right)$ are represented by markers in Fig.6.(a) and the associated curves are plotted in Fig.6.(b). The blue curve results from an arbitrary set of temperatures, leading to high values of $|R|$. The green curve presents critical coupling conditions for mode 3 and quasi critical coupling conditions for mode 2 . The orange curve corresponds to quasi critical coupling conditions for mode 1 . All $\left(T_{1}, T_{2}\right)$ configurations described by points belonging to the segment included between the green and orange points are plotted in gray in Fig.6.(b). The resulting gray bundle shows how $\mathrm{R}$ is scattered if a small variation of control temperatures is considered. The result illustrates the capability of the set-up to control the critical coupling conditions for R. The eABH provides therefore a high controllability of the reflection coefficient. 


\section{Conclusion}

A semi-active $\mathrm{ABH}$ system, called enhanced Acoustic Black Hole (eABH) is proposed: it consists in a classical tapered ABH beam covered with a layer of SMP (Shape Memory Polymer) material, associated to a local multi-points thermal control. Five tunable heating points are used in order to control the profile of the stiffness and damping properties. The result is a deep control of the $\mathrm{ABH}$ reflection coefficient. The capability of the system to reach critical coupling conditions is numerically and experimentally demonstrated. Null reflection property can be achieved at one or several targeted frequencies since critical coupling conditions for several local modes of the ABH termination can be achieved simultaneously. A high level of control for the reflection coefficient is demonstrated, showing the pertinence of the eABH for wave manipulation.

The presented system consists of an open loop in the sense that the temperatures are adjusted and the reflection coefficient is then measured. In future work, this eABH can greatly benefit from a closed loop, where the temperature distribution is controlled to obtain a given reflection coefficient.

Finally, note that another way to control the reflection coefficient of the ABH would be to use a piezoelectric transducer, shunted by an appropriated impedance (resistance and inductance for example). Such a device can be expected to add a significant change to the local complex bending stiffness and, consequently, to the zeros of $\mathrm{R}$ in the complex frequency plane.

\section{Acknowledgments}

The work has been founded by the Agence Nationale de la Recherche in the framework of the project eTNAA (ANR-17-CE08-0035-01, 2017-2021). The authors thanks Benoit Verdin, David Renault and Gaël Matten for the development of the experimental set-up at the applied mechanical department of FEMTO-ST.

\section{References}

[1] A. Pelat, F. Gautier, S. Conlon, and F. Semperlotti. The acoustic black hole: A review of theory and applications. Journal of Sound and Vibration, page 115316, 2020.

[2] J. Deng, O. Guasch, L. Maxit, and L. Zheng. Vibration of cylindrical shells with embedded annular acoustic black holes using the rayleigh-ritz method with gaussian basis functions. Mechanical Systems and Signal Processing, 150:107225, 2021.

[3] J. Deng, O. Guasch, L. Zheng, T. Song, and Y. Cao. Semi-analytical model of an acoustic black hole piezoelectric bimorph cantilever for energy harvesting. Journal of Sound and Vibration, 494:115790, 2021.

[4] J. Cheer, K. Hook, and S. Daley. Active feedforward control of flexural waves in an acoustic black hole terminated beam. Smart Materials and Structures, 30(3):035003, jan 2021.

[5] M. A. Mironov. Propagation of a flexural wave in a plate whose thickness decreases smoothly to zero in a finite interval. Soviet Physics-Acoustics, 34:318-319, 1988.

[6] V.V. Krylov and F.J.B.S. Tilman. Acoustic 'black holes' for flexural waves as effective vibration dampers. Journal of Sound and Vibration, 274(3):605-619, 2004.

[7] J. Leng, V. Romero-Garcia, A. Pelat, R. Pico, J.-P. Groby, and F. Gautier. Interpretation of the acoustic black hole effect based on the concept of critical coupling. Journal of Sound and Vibration, 471:115199, 2020.

[8] K. Hook, J. Cheer, and S. Daley. A parametric study of an acoustic black hole on a beam. The Journal of the Acoustical Society of America, $145,2019$.

[9] V. Romero-Garcia, G. Theocharis, O. Richoux, and V. Pagneux. Use of complex frequency plane to design broadband and sub-wavelength absorbers. The Journal of the Acoustical Society of America, 139(3395), 2016.

[10] J. Leng, F. Gautier, A. Pelat, R. Pico, J.-P. Groby, and V. Romero-Garcia. Limits of flexural wave absorption by open lossy resonators: reflection and transmission problems. New Journal of Physics, 21(5):053003, 2019.

[11] N. Jimenez, V. Romero-Garcia, V. Pagneux, and J.-P. Groby. Rainbow-trapping absorbers: Broadband, perfect and asymmetric sound absorption by subwavelength panels for transmission problems. Scientific Reports, 7(13595), 2017.

[12] V. Denis, F. Gautier, A. Pelat, and J. Poittevin. Measurement and modelling of the reflection coefficient of an acoustic black hole termination. Journal of Sound and Vibration, 349:67 - 79, 2015.

[13] S. Park, M. Kim, and W. Jeon. Experimental validation of vibration damping using an archimedean spiral acoustic black hole. Journal of Sound and Vibration, 459:114838, 2019.

[14] L. Irazu and M. J. Elejabarrieta. A novel hybrid sandwich structure: Viscoelastic and eddy current damping. Materials \& Design, 140:460-472, 2018.

[15] A. Ganguli, A. Deraemaeker, and A. Preumont. Regenerative chatter reduction by active damping control. Journal of Sound and Vibration, 300:847-862, 2007.

[16] A. M. Coppola, A. S. Griffin, N. R. Sottos, and S. R. White. Retention of mechanical performance of polymer matrix composites above the glass transition temperature by vascular cooling. Composites Part A, 78:412-423, 2015.

[17] R.-X. Zhang, Q.-Q. Ni, A. Masuda, T. Yamamura, and M. Iwamoto. Vibration characteristics of laminated composite plates with embedded shape memory alloys. Composite Structures, 74:389-398, 2006. 
[18] P. Butaud, E. Foltête, and M. Ouisse. Sandwich structures with tunable damping properties: On the use of shape memory polymer as viscoelastic core. Composite Structures, 153:401 - 408, 2016.

[19] K. Billon, M. Ouisse, E. Sadoulet-Reboul, M. Collet, P. Butaud, G. Chevallier, and A. Khelif. Design and experimental validation of a temperature-driven adaptive phononic crystal slab. Smart Materials and Structures, 28(3), 2019.

[20] M. Ouisse, D. Renault, P. Butaud, and E. Sadoulet-Reboul. Damping control for improvement of acoustic black hole effect. Journal of Sound and Vibration, 454:63 - 72, 2019.

[21] M. R. Sheperd, P. A. Feurtado, and S. C. Conlon. Multi-objective optimization of acoustic black hole vibration absorbers. The Journal of the Acoustical Society of America, 140, 2016.

[22] L. Ma and L. Cheng. Topological optimization of damping layout for minimized sound radiation of an acoustic black hole plate. Journal of Sound and Vibration, 458:349-364, 2019.

[23] W. Zheng, S. He, R. Tang, and S. He. Damping enhancement using axially functionally graded porous structure based on acoustic black hole effect. Materials, 2019.

[24] W. Huang, H. Zhang, D. J. Inman, J. Qiu, C. E.S Cesnik, and H. Ji. Low reflection effect by 3d printed functionally graded acoustic black holes. Journal of Sound and Vibration, 450:96-108, 2019.

[25] P. Butaud, D. Renault, B. Verdin, M. Ouisse, and G. Chevallier. In-core heat distribution control for adaptive damping and stiffness tuning of composite structures. Smart Materials and Structures, 2020.

[26] D. McCoul, S. Rosset, N. Besse, and H. Shea. Multifunctional shape memory electrodes for dielectric elastomer actuators enabling high holding force and low-voltage multisegment addressing. Smart Materials and Structures, 26, 2016.

[27] V.B. Georgiev, J. Cuenca, F. Gautier, L. Simon, and V.V. Krylov. Damping of structural vibrations in beams and elliptical plates using the acoustic black hole effect. Journal of Sound and Vibration, 330(11):2497 - 2508, 2011. 